\title{
novità in I i breria
}

Anna Costantini, Luigi Grassi, Massimo Biondi

\section{PSICOLOGIA E TUMORI. UNA GUIDA PER REAGIRE}

Un libro di 288 pagine. Lire 30.000

\section{IL LIBRO}

it Cosa significa ricevere una diagnosi di CANCRO?

Q Quali fattori possono attenuare l'IMPATTO PSICOLOGICO?

Ci sono modi "migliori" di altri per AFFrONTARE LA MALATTIA?

Se una persona non ce la fa, si sente DISPERATA, come fare?

È giusto che il paziente sappia che è MALATO di cancro?

Come essere veramente di AlUTO ad una persona cara?

A CHI RIVOLGERSI per avere un aiuto psicologico o psichiatrico?

Quando una persona è depressa o in ansia a causa della malattia, che ruolo hanno gli PSICOFARMACl?

Esistono CURE "ALTERNATIVE" efficaci contro il cancro?

C'è un MODO MIGLIORE per dire a un malato che ha poco da vivere?

Cosa sono le TERAPIE PALLIATIVE?

Una volta GUARITI, che conseguenze psicologiche lascia il trauma del cancro?

\section{GLI AUTORI}

ANNA COSTANTINI, 42 anni, psicologa e psicoterapeuta presso il Dipartimento di Salute Mentale della ASL Roma D, da anni si occupa di Psicologia Oncologica e di Psicoterapia di gruppo ed è docente in vari corsi pubblici di formazione.

LUIGI GRASSI, 41 anni, psichiatra, Vice Presidente della SIPO, è docente di Psichiatria e coordinatore del Servizio di Psichiatria di Consultazione e Collegamento presso l'Università di Ferrara.

MASSIMO BIONDI, 46 anni, psichiatra presso la $3^{\text {a }}$ Clinica Psichiatrica dell'Università "La Sapienza" di Roma, è Vice Presidente della Società Italiana di Medicina Psicosomatica, ha pubblicato numerosi volumi su temi di Psicopatologia, Psicosomatica e Psiconeuroimmunologia. 


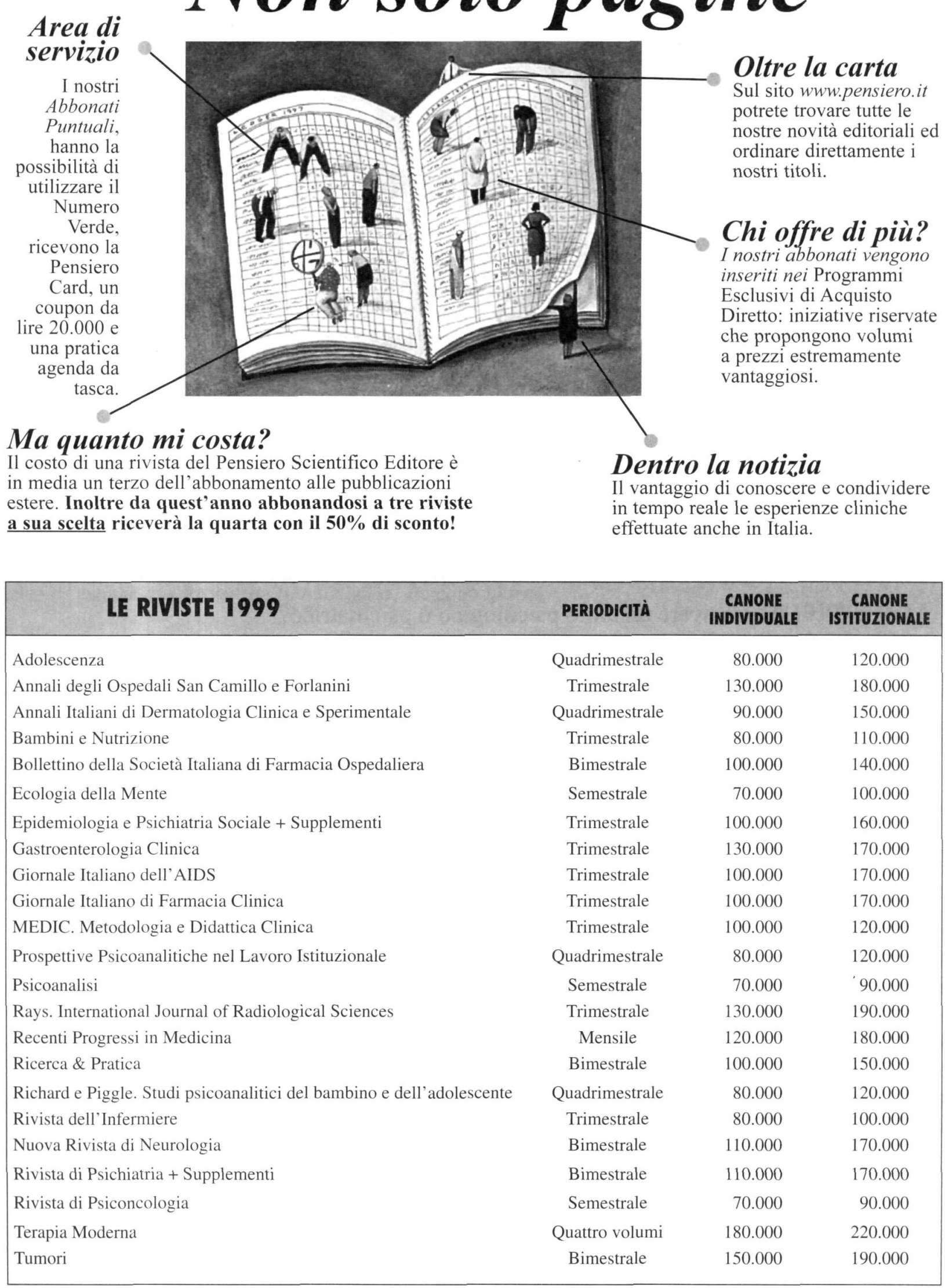

CHIAMI IL NOSTRO NUMERO VERDE 167-259620 PER CONCORDARE IL SUO ABBONAMENTO PERSONALIZZATO

Il Pensiero Scientifico Editore. Libri e riviste, nostri contemporanei 


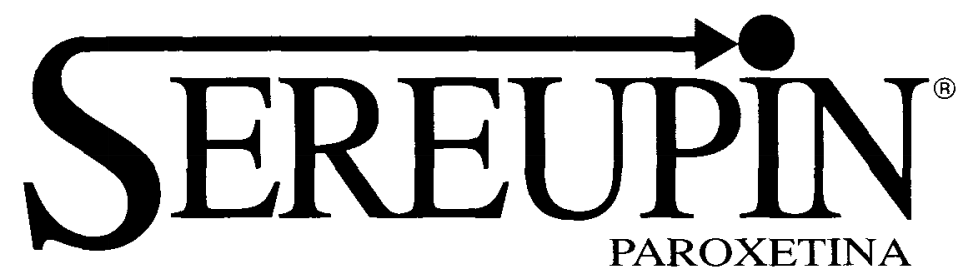

RIASSUNTO DELLE CARATTERISTICHE DEL PRODOTTO

1. DENOMINAZIONE DELLA SPECIALITA MEDICINALE SEREUPIN.

2. COMPOSIZIONE QUALTTATVA E QUANTITATIVA Una compressa rivestita divisibile contiene

Principio attivo:

Paroxetina cloridrato $22,88 \mathrm{mg}$

corrispondente a paroxetina $20,00 \mathrm{mg}$

3. FORMA FARMACEUTICA Compresse rivestite divisibili

4. INFORMAZIONI CLINICHE 4.1 Indicazioni terapeutiche SEREUPIN è indicato nel trattamento della depressione di tutti i tipi, compresa la depressione accompagnata da ansietà e nella prevenzione delle recidive e delle ricadute della depressione. SEREUPIN è indicato nel trattamento del disturbo ossessivo compulsivo e nella prevenzione delle sue recidive. SEREUPIN è indicato nel trattamento del disturbo da attacchi di panico con o senza agorafobia. L'aggiunta di paroxetina alla terapia cognitiva di tipo comportamentale si è dimostrata significativamente piu efficace nel trattamento del disturbo da attacchi di panico rispetto alla terapia coonitiva comportamentale da sola. 4.2 Posologia e modo di somministrazione - Depressione La dose raccomandata è di $20 \mathrm{mg}$, una volta al giorno. II dosaggio puo essere aumentato fino a $50 \mathrm{mg}$ al giorno. in base alla risposta del paziente, con aumenti graduali di $10 \mathrm{mg}$. Disturbo ossessivo compulsivo La dose raccomandata è di $40 \mathrm{mg}$ al giorno. La dose iniziale è di $20 \mathrm{mg}$ al giorno e puo essere aumentata settimanalmente con aumenti graduali di $10 \mathrm{mg}$ in base alla risposta del paziente. in alcuni casi il dosaggio può essere aumentato fino ad un massimo di $60 \mathrm{mg}$ al giorno. - Disturbo da attacchi di panico La dose raccomandała è di $40 \mathrm{mg}$ al giorno. La dose iniziale e di $10 \mathrm{mg}$ al giorno e può essere aumentata settimanalmente con aumenti graduali di $10 \mathrm{mg}$ in base alla risposta del paziente. Un basso dosaggio iniziale è raccomandato per ridurre al minimo il potenziale peggioramento della sintomatologia da panico, come si è osservato generalmente nel trattamento iniziale del disturbo da attacchi di panico. Come per tutti i farmaci antidepressivi, il dosaggio, in base alla risposta terapeutica, deve essere aggiustato e rivisto se necessario entro le prime due o tre settimane dall inizio della terapia. Nell'anziano, visti i risultati degli studi farmacocinetici, è consigliabile un dosaggio di $20 \mathrm{mg}$ al giomo. In alcuni pazienti può essere necessario aumentare la dose: questo deve comunque awenire gradualmente con incrementi di $10 \mathrm{mg}$ alla volta fino ad un massimo di $40 \mathrm{mg}$ al giorno in accordo alla risposta del paziente. In pazienti con insufficienza renale grave (clearance della creatinina $30 \mathrm{ml} / \mathrm{min}$.) o insufficienza epatica grave si verifica un aumento delle concentrazioni plasmatiche di paroxetina, pertanto si raccomanda la somministrazione del dosaggio minimo previsto. I pazienti dovrebbero essere trattati per un periodo sufficiente da assicurare la remissione completa dei sintomi; tate periodo puo essere di motti mesi sia per la depressione, sia per il disturbo ossessivo compuisivo e per il disturbo da attacchi ci panico. 4.3 Controindicazioni Ipersensibilità verso i componenti o verso altre sostanze strettamente correlate dai punto di vista chimico. Non somministrare in età pediatrica. Generalmente controindicato in gravidanza $e$ durante f'allattamento. 4.4 Speciali avvertenze e precauzioni per l'uso Come nella maggior parte delie terapie con antidepressivi, SEREUPIN non dourebbe essere somministrato in associazione con anti-MAO 0 prima di due settimane dal termine di tale trattamento. Analogamente non è opportuno iniziare una terapia con anti-MAO prima di due settimane dai termine della terapia con paroxetina. SEREUPIN non produce variazioni cliniche significative della pressione arteriosa, della trequenza cardiaca e dell ECG, ciononostante occorre osservare le consuete precauzioni in presenza di atterazioni cardiovascolari. Come nel caso di attri antidepressivi, SEREUPIN dovrebbe essere usato con cautela in pazienti epilettici. Complessivamente l'incidenza di convulsioni in pazienti trattati con SEREUPIN è stata <0,1\%. I| trattamento con SEREUPIN deve essere sospeso nei pazienti che presentino convulsioni. SEREUPIN dovrebbe essere usato con precauzione in pazienti con storia clinica di mania. Particolare attenzione occorre nell'uso di SEREUPIN nei pazienti in trattamento con anticoagulanti orali. Studi condotti con cimetidina hanno dimostrato che quest'utima determina un aumento della biodisponibilità di paroxetina di circa il $45 \%$, pertanto si raccomanda límpieco di SEREUPIN al dosaggio minimo. Esiste un'espenienza clinica limitata nella somministrazione concomitante di paroxetina con ECT. Quando si interrompe bruscamente il trattamento con inibitori della ricaptazione della serotonina possono comparire: insonnia, vertigini, sudorazione, palpitazioni, nausea, ansia, irrtiabilità, pare stesie e cefalea. Pertanto, quando si decide di interompere il trattamento, le dosi devono essere diminuite in modo graduale per ridure al minimo l'entità di tali sintomi. Porre attenzione a non interpretare tali sintomi attribuendoli ad un peggioramento della malattia psichiatrica trattata. Tenere fuori dalla portata dei bambini. 4.5 Interazioni con altri medicamenti e altre forme di interazione Specifici studi sono stati condotti per valutare le possibili interazioni tra paroxetina e farmaci o sostanze psicoattive. L'esperienza in un limitato numero di soggetti sani ha dimostrato che paroxetina non aumenta la sedazione e la debolezza associate ad aloperidolo, amobarbital od oxazepam, quando somministrati contemporaneamente. Gli studi condotti nell'animale indicano che può verificarsi una interazione tra paroxetina e inibitori delle monoaminossidasi (antiMAO) o triptofano, analogamente ad altri inibitor della ricaptazione di serotonina, e che può determinare in tali circostanze la "sindrome da serotonina" caratterizzata da agitazione, irequietezza e sintomi gastrointestinali quali diarrea. In uno studio in pazienti depressi stabilizzati con litio, non si è osservata interazione farmacocinetica tra paroxetina e litio. Dato che l'esperienza clinica è limitata, occorre particolare attenzione nella somministrazione contemporanea di paroxetina e litio. La co-somministrazione di paroxetina e fenitoina è associata ad una diminuzione nella concentrazione plasmatica di paroxetina. Quando questi due farmaci sono co-somministrati non è necessario un aggiustamento della dose iniziale con paroxetina, ogni eventuale aggiustamento della dose dovia essere valuntato sulla base della risposta clinica. L'assunzione con anticonuusivanti può essere associata ad un aumento dell 'incidenza di reazioni indesiderate. Sebbene la paroxetina non aumenti gli effetti dannosi psicomotori indotti dalla assunzione di alcool, il bro uso concomitante è sconsigliato. Come con altri antidepressivi, inclusi gli SSRI, SEREUPIN puod, a livello del citocromo P450, interterre sul metabolismo epatico di alcuni tarmaci determinandone laumento dei livelli plasmatici, fra questi: debrisoctina, sparteina, alcuni antidepressivi triciclici, neurdettici, tenotiazinici e gli antiantmici di classe $1 \mathrm{C}$. II metabolismo e la farmacocinetica di SEREUPIN possono essere influenzati dai farmaci che provocano una induzione 0 una inibizione del metabolismo enzimatico. Nel caso in aui SEREUPIN sia somministrato contemporaneamente a farmaci inibitori del metabolismo enzimatico si suggerisce l'uso dei dosaggi più bassi. Nel caso di co-somministrazione contemporanea a farmaci induttori del metabolismo enzimatico non è richiesto alcun aggiustamento iniziale del dosaggio. Ogni successivo aggiustamento deve essere basato sulta risposta dinica. Una interazione fammacocinetica significativa è stata evidenziata anche tra paroxetina e prociclidina con aumento dei livelli plasmatici di quest ultima quando somministrate contemporaneamente. Se si osservano effetti anticolinergici, la dose di prociclidina dovrebbe essere ridotta. Dati preliminari suggeriscono una possibile interazione farmacodinamica tra paroxetina e warlarina che puo compottare un allungamento del tempo di emorragia in presenza di valori normali di protrombina. 4.6 Gravidanza e allattamento Nonostante gli studi negli animali non abbiano dimostrato alcun effetto teratogeno od embriotossico selettivo, la sicurezza della paroxetina nella donna in gravidanza non è stata stabilita; pertanto il prodotto non dovrebbe essere utilizzato durante la gravidanza o lailattamento se non nei casi in cui il potenziale beneficio superi il possibile rischio e comunque sotto diretto controllo medico. 4.7 Effetti sulla capacità di guidare e sull'uso di macchinari L'esperienza clinica ha dimostrato che la terapia con paroxetina non è associata ad alterazioni delle funzioni cognitive o psicomotorie. Tuttavia. come con tutti i farmaci psicoattivi, i pazienti dovrebbe ro essere awertiti di usare cautela nell'uso di macchinari pericolosi e nella guida di veicoli. 4.8 Effetti indesiderati Gli effetti indesiderati con paroxetina sono di moderata entità e non influenzano la qualità di vita del paziente; generalmente non richiedono interruzione della terapia e durante il trattamento prolungato possono diminuire di intensità e freouenza. Le reazioni awerse associate all'uso di paroxetina piu comunemente osservate sono: nausea, sonnolenza, sudorzzione, tremore, astenia, secchezza delle fauci, insonnia, distunzioni sessuali, vertigini, costipazione, diarrea e diminuzione dell'appetito. Raramente sono state riscontrate convulsioni. Occasionalmente sono stati riportati disturbi extrapiramicali (distonia oro-facciale) in pazienti con pregressi disturbi del movimento o in pazienti in trattamento con neurolettici, prevalentemente nell'anziano e stata riscontrata iponatriemia, che generalmente scompare con la sospensione del farmaco. Sono stati riscontrati raramente transitori aumenti degli enzimi epatici. Sebbene non sia stata dimostrata una relazione causale con paroxetina, si consiglia di sospendere it trattamento in caso di comparsa di alterazioni della funzionalità epatica. Paroxetina, rispetto agii antidepressivi tricicicici, è associata ad una minore probabilità di causare secchezza delle fauci, costipazione e sonnoienza. 4.9 Sovradosaggio I sintomi di sovradosaggio con paroxetina includono nausea, vomito, tremore, midriasi, secchezza deile fauci e irritabilita. Non si sono osservati casi di anormalità nell'ECG. coma o convulisioni successive al sovradosaggio con paroxetina. Casi di sovradosaggio sono stati segnalati con paroxetina fino a $2000 \mathrm{mg}$, da soli o in associazione con altri farmaci Quando usato da solo, SEREUPIN non ha mai causato morte e il recupero del paziente non ha lasciato sequele. II trattamento si basa sulle abituali misure utilizzate nel sovradosaggio con antidepressivi: si consiglia svuotamento gastrico attraverso induzione di emesi o lavanda gastrica. Successivamente può essere somministrato carbone attivo, 20030 grammi ogni $4-6$ ore nelle prime 24 ore dopo ingestione. Ė indicata una terapia di supporto con attenta osservazione e frequente monitoraggio dei sintomi vitali. La precoce somministrazione di carbone attivo può ritardare l'assorbimento di SEREUPIN. 5. PROPRIETÁ FARMACOLOGICHE 5.1 Proprietà farmacodinamiche La paroxetina è una molecola con una azione inibitoria potente e selettiva sulla ricaptazione della serotonina (5-idrossitriptamina: $5 \mathrm{HT}$ ) nei neuroni cerebrali, senza interferenze sulla captazione della noradrenalina. La sua efficacia nei trattamenti della depressione, del disturbo ossessivo compulsivo e dei disturbo da attacchi di panico è presumibilmente correlata a tale meccanismo. Chimicamente è una (-)-trans-4-(4' fluorctenil)-3-(3', 4' metilendiossi-fenossimetil)-piperidina cioridrato, la cui struttura non è riconducibile a quella degli antidepressivi triciclici, tetraciclici e di altri disponibili. Negil studi a iungo termine con paroxetina è emerso che lefficacia si mantiene per periodi di almeno un anno nel trattamento della depressione e del disturbo ossessivo compulsivo e per oitre un anno nel trattamento del disturbo da attacchi di panico. 5.2 Proprietá farmacocinetiche La paroxetina è ben assorbita nel tratto gastrointestinaie dopo somministrazione orale. I livelli sistemici de equilibrio sono raggiunti entro 7-14 giorni dall 'inizio del trattamento. Circa il $95 \%$ della paroxetina presente nel plasma è legato alle proteine. L'emivita di eliminazione è generalmente di un giorno. La paroxetina e metabolizzata a livello epatico: gil studi di farmacologia hanno evidenziato che i metaboliti sono molto meno potenti della paroxetina. pertanto si presume che non contribuiscano al suo effetto terapeutico. I principali metaboliti sono prodotti polari e coniugati, prontamente eliminati. L'escrezione urinaria di paroxetina e circa il $64 \%$ della dose di cui meno del $2 \%$ in forma immodif. cata. Circa il $36 \%$ della dose è escreto nelle feci, attraverso la bile, di cui la forma immodificata raporesenta meno dell' $1 \%$. II profilo farmacocinetico non sembra modificarsi durante la terapia a lungo termine. La biodisponibilità della paroxetina non è influenzata dalla contemporanea assunzione di cibo. Nei soggetti anziani le concentrazioni plasmatiche di paroxetina sono risultate elevate. 5.3 Dati preclinici di sicurezza Gli studi di tossictà acuta hanno evidenziato valori di $L_{50}$ pari a 374 e $341 \mathrm{mg} / \mathrm{kg}$ dopo somministrazione orale e valori pari a 27 e $38 \mathrm{mg} / \mathrm{kg}$ dopo somministrazione e.v. rispettivamente nel ratto e nel topo. Negli studi di tossicità per somministrazioni ripetute nel ratto è stato evidenziato un lieve incremento dei valori di fosfatasi alcalina a dosi pari a $4 \mathrm{mg} / \mathrm{kg} / g i o m o$ e di alaninaminotransferasi alle dosi di 12 o $40 \mathrm{mg} / \mathrm{kg} / \mathrm{giomo}$. Come atteso per le ammine lipofile, inclusi gil antidepressivi triciclici, è stata riscontrata fosfolipidosi nel ratto, ma non e stata osservata invece negli studi condotti ne primati della durata di un anno a dosi superiori a 6 volte il dosaggio clinico raccomandato. Nella scimmia Rhesus è stato evidenziato un aumento dei lisosomi nel fegato a dosi elevate dopo 12 mesi di trattamento. In questa specie la dose che non ha causato effetti tossici è stata di 1 $\mathrm{mg} / \mathrm{kg} / \mathrm{giorno}$. Non è stata evidenziata teratogenictà nei rattio e nel coniglio alle dosi tossiche materne, mentre una embriotossicità non selettiva viene segnalata agli stessi livelli di dose. Nel ratto gli studi sulla fertilità hanno evidenziato alla dose di $13 \mathrm{mg} / \mathrm{kg}$ una trequenza ridotta delle gravidanze e, a $43 \mathrm{mg} / \mathrm{kg} /$ giorno una minore sopravivenza post-partum dei nati. Dosi pari a $50 \mathrm{mg} / \mathrm{kg}$ giorno hanno causalo effetti tossici sul ciclo estrale e sull'ovulazione, nei maschi invece sono state evidenziate variazioni istopatologiche a carico della rete testis e dell'epididimo. Nessun potenziale cancerogeno è stato riscontrato negli studi, della durata di due anni, condotti nel ratto e nel topo e nessun effetto genotossico è stato osservato negli studi di mutagenesi in vitro ed in vivo. 6. INFORMAZIONI FARMACEUTICHE 6.1 Elenco degli eccipienti Calcio fosfato bibasico biidrato, idrossipropilmetilcellulosa, magnesio stearato, polietilenglicole 400 , polisorbato 80 (E 433), sodio carbossimetilamido, titanio biossido (E 171). 6.2 incompatibilità Nessuna. 6.3 Validità 2 anni. 6.4 Speciali precauzioni per la conservazione Nessuna. 6.5 Natura e contenuto del contenitore Astuccio contenente un blister (PVC/AI oppure PVC/PVDC/Al) da 12 compresse rivestite divisibli di $20 \mathrm{mg} .6 .6$ Istruzioni per I'uso Nessuna. 7. TITOLARE DELL'AUTORIZZAZIONE ALL'IMMISSIONE IN COMMERCIO Ravizza Farmaceutici Spa - Via Europa, 35 - 20053 Muggio (Milano). 8. NUMERO DELL'AUTORIZZAZIONE ALL'IMMISSIONE IN COMMERCIO A.I.C.: $n$. 027965019. 9. DATA DI PRIMA AUTORZZAZIONE/RINMO VO OELL'AUTORIZZAZIONE Prima autorizzazione: 01.02.1993. Rinnovo autorizzazione: 16.02.1998. 10. TABELLA DI APPARTENENZA SECONDO IL DPR 9 OTTOBRE 1990, N. 309 Nessuna. 11. REGIIME DI DISPENSAZIONE AL PUBBLICO La vendita al pubblico è subordinata aila presentazione di ricetta medica. 12. DATA DI (PARZIALE) REVISIONE DEL TESTO Agosto 1998.

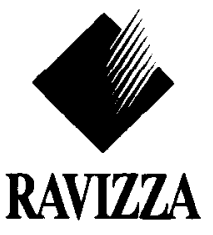




\section{Mark Spivak (1929-1998).}

In memoriam, L. Burti

I

\section{Editoriali}

Epidemiological aspects of some problems in child and adolescent psychiatry, D. Shaffer 151

Developmental psychopathology: a framework for planning child mental health,

$$
\text { D.J. Cohen, E. Caffo }
$$

156

The epidemiology of child and adolescent psychiatric disorders: recent developments and issues, E. Fombonne

161

Hyperactivity and its primary disorders in the childhood population, $P$. Hill

167

\section{Articoli}

T. Tomov, Multiple perspectives on the evaluation of outcome in Eastern Europe

173

L. Magliano, A. Fiorillo, C. Malangone, A. Aletti, G. Belotti, P. Bevilacqua, A.L. Delle Femine, G. Fontana, F. Maucioni, M. Travi, P. Zanus, A. Rossi, M. Maj,

Carico familiare nella schizofrenia: effetto delle variabili cliniche e socio-ambientali e degli interventi familiari

178

R. Madonna, M. Valenti, G. Borrelli, R. Cerbo, M. De Lellis, G. Sprovera, M. Massaro, A. D'Alessandro, M. Contina Marinucci, S. Tiberti, F. Di Orio, Osservazione epidemiologica degli handicap

psiconeurosensoriali nella popolazione abruzzese (0-24 anni). Primi risultati

188

M. Percudani, M. Knapp, La prospettiva economica nell'assistenza e nel trattamento dei pazienti con diagnosi di schizofrenia

197

A. Saltini, D. Cappellari, P. Cellerino, L. Del Piccolo, Ch. Zimmermann,

Uno strumento per la valutazione dell'intervista medica nel contesto della medicina generale:

il VR-MICS/D (Verona-Medical Interview Classification System/Doctor)

210

Società Italiana di Epidemiologia Psichiatrica (SIEP)

Riunione Scientifica Annuale (Bari, 20 novembre 1998)

224

Indice generale del volume 7 (1998)

226

Lire 40.000 